\title{
A new hybrid nature-inspired algorithm for multi-objective engineering optimization
}

\author{
Zhijie Zhang \\ College of Computer Sci \& Tech, \\ Southwest University for Nationalities, \\ Chengdu Sichuan, China, 610041 \\ e-mail: cdznf@126.com
}

\begin{abstract}
-in this paper a new natured-inspired algorithm, based on the based on the prevalent mechanism of infectious diseases, was designed for multi-objective optimization. This optimization algorithm derived from the susceptible infected and removed model, mimic the transmission mechanism of infectious diseases, called hybrid infection model optimization algorithm (HIA). Similar to the other natured-inspired algorithms, the HIA used the spread transmission way of disease and the incremental iteration movement of the every generation new sources of infection to search the global optimum. The results showed that the HIA has good convergence and can quick search to the global optimal solution with fewer time of iterations for multi-objective optimizations. For some optimization instances, the HIA algorithm has faster speed for search better approximate solution or search the best solution more precisely.
\end{abstract}

Keywords-natured-inspired; optimzation; hybrid; multi-objective; infectious mechanism

\section{INTRODUCTION}

The process of optimization often is that using some methods to search the best or the worst solution or some specific max/min objects in special target space. The goal of optimization is found the better fitting solution under some constraint conditions. All of these optimization questions have very important values for theory research and application research. According to non-linear constraint conditions, the optimization question with exactly objects often could be defined as following [1][2]: $\underset{x \in \Re^{n}}{\max \text { imize }}$ imize : , $f_{i}(x), x=\left(x_{1}, x_{2}, \ldots, x_{n}\right)^{T} \in \mathfrak{R}^{n}$,

Subject to:

$\phi_{j}(x)=0,(j=1,2, . ., M)$, $\psi_{k}(x) \geq 0,(k=1,2, \ldots, N)$

The $f(x) 、 \phi_{i}(x)$ and $\psi_{j}(x)$ in the expression of $(1.1)(1.2)(1.3)$ stand for the scalar functions of row vector. The $x_{j}$ in $x=\left(x_{1}, x_{2}, \ldots, x_{n}\right)^{T}$ could be called the design variables, or the main variables, or the dominant variables, in this paper the $x_{j}$ were unify called as the main variable/ variables $\mathrm{s}$.

Every main variable $x_{j}$ could be continue variable, discrete variable or hybrid variable with discreteness character and consecutiveness character. To every main variable $x_{j}$, it belong to vector $x$, and always lie in n-dimension space $\mathfrak{R}^{n}$. The function $f(x)$ was called the cost function or objective function, The following in this paper use the definition of objective function mainly stand for non-linear function.

For most of optimization problems, nature-inspired mix other meta-heuristics are a group of better method [3][4]. The new nature-inspired optimization algorithm, hybrid meta-heuristic tech, could quickly obtained the approximately solutions that meet the requirement of accuracy and meet the requirement of computing time, could solve the NP-hard problem and get the approximate solution, could solve a variety of difficultly hard-optimization problem and get the approximate solution on time [5].

The new hybrid optimization algorithm has better ability to solve nonlinear problem, has better adaptability to solve dynamic parameter problem [6][7]. The new nature-inspired optimization algorithm is easy to hybrid with other optimization tech or method and is easy to use [8][9][10]. So the new nature-inspired algorithm can be used to solve the NP-hard problem or hard-optimization problem, for application of optimization algorithm, has the important of research.

The main research direction and research work of this article, is based on the types and features of the problem needed to be solved, use the model of susceptible infected 
and removed model(SIR), with the advantage of nature-inspired algorithm synthetically, to achieve a better global and local search strategy of new nature-inspired algorithm by hybrid tech. The new hybrid algorithm should be do some experiments and compare to with other algorithm for the application and research.

\section{NATURE-INSPIRED}

With the emergence of the genetic algorithm, the modern nature-inspired algorithm, as a kind of new meta-heuristic search method, has come into being in 1972. The nature-inspired algorithm is presented the characteristics of the hybrid algorithm from begin, it is easy to solve the complex optimization problem that hard to deal with by the method of traditional optimization tech [11][12], such as engineering optimization problem, business planning problem, course arrangement, TSP, MCP, path planning problem etc.

For its superior performance, so the nature-inspired method is widely used in various kinds of optimization problems by hybrid method. At the same time, more and more new hybrid optimization algorithms based on nature-inspired search method emerge at the historic moment, such as: Simulated Annealing algorithm [13], Particle Swarm Optimization algorithm [14], Ant Colony Optimization algorithm [15], Harmony Search algorithm [16], Cuckoo Search algorithm [17], etc. All of them provide more new and better methods to solve more diverse difficultly hard-optimization problems.

The thought of hybrid optimization algorithm based on nature-inspired, in essence, is a kind of mathematical model being inspired by how things survive in nature. The mathematical model reflects the basic ability or characteristic that the nature acquired through competition for survival and the process of evolution in a way of biological mathematical function, such as parallel processing, self-learning, special association, pattern recognition, classification, episodic memory [18], etc.

Then, the math model of new algorithm should be converted into search and optimization algorithm step by step. In the end, the Hybrid Infection-based Nature-Inspired Algorithm was proposed and tested. The next, the design and implementation method of a new Hybrid search algorithm based on nature-inspired was introduced, the kind of Hybrid natural heuristic search algorithm based on infectious disease model, was named
Hybrid Infection-based Nature-Inspired Algorithm(HIA).

\section{HYBRID MODEL}

At present, in the area of mathematical biology, the model of infectious diseases has been researched for many years.

On the one hand, the model for a variety of infectious diseases, combined with the historical statistical data, more statistical analysis had been done, On the other hand, for the establishment of mathematical models and prediction of infectious diseases, more and more mathematical methods were introduced.

The SIR math model should be converted into search and optimization algorithm step by step. The SIR model of discrete time infectious diseases was choose as the prototype by observation, based on the basic natural phenomenon, extracts the corresponding global search algorithm model [19][20].

According to the type of the problem needed be solved, determine the main apply areas that the algorithm need to use, all these of the algorithm of architecture, steps and judgment conditions etc., should be designed, in the end, the Hybrid Infection-based Nature-Inspired Algorithm was proposed and tested.

There are mathematical constraint conditions of hybrid optimization algorithm, as follows [21][22][23]:

$$
\begin{aligned}
& S_{t+1}=S_{t}+b\left(I_{t}+R_{t}\right)-I_{t} S_{t} \beta / N \\
& I_{t+1}=I_{t}(1-\gamma-b)+I_{t} S_{t} \beta / N \\
& R_{t+1}=R_{t}(1-b)+\gamma I_{t}
\end{aligned}
$$

In order to further simplify the new algorithm model, more constraint conditions should be added:

$S_{0}>0, I_{0}>0, R_{0}>0$, and, $S_{0}+I_{0}+R_{0}=N$;

$S_{t}>0, I_{t}>0, R_{t}>0$ and, $S_{t}+I_{t}+R_{t}=N$;

$S_{t+1}>0, I_{t+1}>0, R_{t+1}>0$ and, $S_{t+1}+I_{t+1}+R_{t+1}=N$;

$\beta I_{0} / N \leq I_{0} / N \leq 1$.

For the relationship $S_{t}+I_{t}+R_{t}=N$, so $R_{t}=N-S_{t}-I_{t}$ at any time, then the three formulas could be simplified into four new relations function expression as following:

$$
\begin{aligned}
& S_{t+1}=S_{t}+b\left(I_{t}+R_{t}\right)-I_{t} S_{t} \beta / N \\
& S_{t+1}=S_{t}+b\left(N-S_{t}\right)-I_{t} S_{t} \beta / N=f\left(S_{t}, I_{t}\right) \\
& S_{t+1}=f\left(S_{t}, I_{t}\right) \\
& I_{t+1}=I_{t}(1-\gamma-b)+I_{t} S_{t} \beta / N=g\left(S_{t}, I_{t}\right)
\end{aligned}
$$

In the above formulas, the $S$ stand for the susceptible parts, the I stand for the infected parts and the R stand for the removed parts. The $t$ means counter for time of different parts to every iteration. The $\mathrm{N}$ is the total number of target sample space, and it is a constant number for a constant optimization problem. The $\beta S / N$ stand for transfer probability from $\mathrm{S}$ to $\mathrm{I}$, 
The $\gamma$ stand for transfer probability from $\mathrm{I}$ to $\mathrm{R}$. The $\beta$ stand for contact coefficient, and the contact transmission coefficient is $\Re_{0}$.

\section{HIA HYBRID ALGORITHM}

For design and implement the hybrid nature-inspired optimization algorithm(HIA) algorithm, more works need be done. At first, analyze and compare the spread transmission way of disease and the incremental iteration movement of the every generation new sources of infection. According to the way of construct hybrid search, the HIA algorithm based on the mathematical modeling of SIR, and then hybrid use the tech of meta-heuristic method, mixed implement the global and local search strategies, combined with different techniques of optimization, so a new hybrid search algorithm HIA was designed.

The other needed determined parameters: the total period of infection $\mathrm{T}$; the total sample of Group $\mathrm{N}$; the total number of susceptible individuals $S_{\text {num }}$; the total number of infected individual $I_{\text {num }}$; The total number of individuals with immunity mechanic $R_{\text {num }}$; Contact coefficient $\beta$; the coefficient of was infected and after contact $\gamma$; Contact transmission coefficient $\mathfrak{R}_{0}$; ratio of Leave and join the total group of individual $b$. For initial value of each parameter, depending on the type of optimization problem be initialized. The total period of infection $\mathrm{T}$, stand for total cycles times operated by HIA Algorithm, is experience parameters depending on the type of problem , by the calculate method $T=$ Counter $=1 / \Delta_{i}$ and was set as 100 times .

The total sample of Group $\mathrm{N}$ was set as 1000 , decided by the scope of the solution space. at first, $S_{\text {num }}=N 、 \mathrm{I}_{\text {num }}=0, \mathrm{R}_{\text {rnum }}=0$ then the next generation the $S_{\text {num }} 、 I_{\text {num }}$ and $R_{\text {num }}$, depended on other parameters $\beta$ 、 $\gamma 、 \mathfrak{R}_{0}$ and $\mathrm{b}$, other init set value of HIA algorithm as following: $\gamma=1 、 b=0 、 \beta=\mathfrak{R}_{0} 、 \mathfrak{R}_{0}=3 、 \alpha=0.3$.

The pause code of HIA is list in Figure. 1

\section{HIA hybrid optimization algorithm}

\section{Begin}

objective function $f(x), \quad x=\left(x_{1}, x_{2}, \ldots \ldots, x_{n}\right)^{T}$ initialize the $\mathrm{S} 、 \mathrm{I} 、 \mathrm{R}$ population:

initialize best solution-set $\mathrm{N}$; initialize parameters : $\beta, \gamma, \mathrm{b} 、 \alpha$; initialize iteration counter $\mathrm{c}$; initialize iteration increment $\Delta i$; While $\left(b \beta>(\gamma+b)\left(b-b \gamma-b^{2}\right)\right.$ or $(\mathrm{c}<\max$ number of iterations $))$ Generate new solutions; if $(f(x)$ is better than $\mathrm{N})$ update the best solution-set $\mathrm{N}$

else

Generate new solutions;

\section{end if}

renew all $\omega_{s}=i_{s}-\Delta_{i} 、 \omega_{i}=i_{i}+\alpha \Delta_{i}$ 、

$\omega_{r}=r_{i}+(1-\alpha) \Delta_{i} ;$

renew all $\beta, \gamma, \mathrm{b} 、 \alpha$;

end while

end

Figure 1. pause code of the HIA

The original dynamic constraints of HIA algorithm $0<\beta b\left(1-1 / \Re_{0}\right)<b \Re_{0}$ could be carried out by analyzing the following Jacobian matrix, if the limiting conditions of Jacobian matrix $b \beta>(\gamma+b)\left(b-b \gamma-b^{2}\right)$ could be meet, then, the HIA algorithm always could found appropriate solution. At the same time, another terminal condition decided by the variable of the total period of infection $\mathrm{T}$. The most simple method of terminal condition could designed by this formula: $T=$ Counter $=1 / \Delta_{i}$.

Another terminal condition is that the counter of HIA had reached predetermined times of iterations, or, the optimal solution of HIA algorithm without any improvement after the set times of iterations had been reached.

The hybrid infection model optimization algorithm (HIA) based on natured-inspired mechanic, using theory of two-dimensional brownian motion as random walking mechanism. The random walk should be used in the HIA, the essence is one kind of candidate solution generation mechanic in solution space. Its concept is an ideal mathematical brownian motion state and could be represented by the brownian motion. The two-dimensions brownian motion was used as HIA global search strategy to generate the initial set of candidate solutions to gain every generation of random variable.

The brownian motion was used as one-dimensional motion formula, then expanded it to two-dimensional motion formula.

The mean square offset formula of one-dimensional brownian motion : $\quad<x^{2}>=2 D t$. The mean square offset formula of 2-dimensions brownian motion: $r_{2}^{2}=4 D t=4 k_{B} T t / 6 \pi \eta \alpha$.

In the $\mathrm{f}$ mean square formula, $\mathrm{D}$ is the diffusion coefficient $D=k_{B} T / u, \mathrm{~T}$ is the temperature, $k_{B}$ is the absorption coefficient , $u$ is stokes coefficients $u=6 \pi \eta \alpha, \quad \alpha$ is the particle radius, $\eta$ is the coefficient of viscosity, $\mathrm{t}$ stand for time. 


\section{ENGINEERING OPTIMIZATION}

For the objective function $f(x)$ of HIA were defined as $x=\left(x_{1}, x_{2}, \ldots \ldots, x_{n}\right)^{T}$, the HIA algorithm could be used as tool to deal with multi-objective engineering optimization problem.

For the testing of HIA algorithm, the spring design optimization problem and the welded beam design problem were used as test problems.

The Spring Design Optimization Problem [24], Mainly in order to solve the different between the three variables of multi-objective optimization problem: the length of the spring L; the spring wire radius w; the radius of the spring $\mathrm{d}$.

The spring design goal are to minimize the weight of the spring, to minimize the bias of spring and at the same time, to maximize the shear stress in various constraints, to meet the requirements of the spring of a variety of physical shape, etc. The spring design optimization problem can be expressed in the following mathematical equations as follows:

Minimise :

$f(x)=(L+2) \times w \times d^{2}$

subject to:

$g(x)=1-\frac{d^{3} L}{7178 \times w^{4}} \leq 0$

$g(x)=1-\frac{140.45 w}{d^{2} \times L} \leq 0$

$g(x)=\frac{w \times d}{1.5}-1 \leq 0$

$g(x)=\frac{4 d^{2}-d \times w}{12566 \times d \times w^{3}-w^{4}}+\frac{1}{5108 \times w^{2}}-1 \leq 0$

The parameters of above mathematical equations meet the constraints as follows:

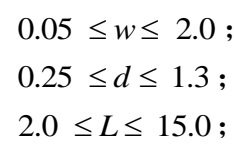

By HIA the result could be found is $f_{*}=0.012665$, the exactly value of parameters are $w=0.051690, d=0.356750, L=11.287126$ in average less than 60 iterations..

Another famous engineering optimization problem is the welded beam design problem [25]. The multi-objective optimization problem has four constraints: $\mathrm{w}$ - the width of the welding beam welding area, $\mathrm{L}$ - the length of the welding beam welding area, $d$ - the width of the welding beam $\mathrm{d}$ and $\mathrm{h}$ - the height of the welding beam.

The parameters of welded beam design are defined as follows:

$\sigma(x)=\frac{504000}{h d^{2}}$ $\delta(x)=\frac{65856000}{\left(30 \times 10^{6}\right) \times h \times d^{3}}$

$P(x)=\frac{4.013\left(30 \times 10^{6}\right) \sqrt{\frac{d^{2} h^{6}}{36}}}{196}\left(1-\frac{\left.d \sqrt{\frac{30 \times 10^{6}}{4\left(12 \times 10^{6}\right)}}\right)}{28}\right)$

$\tau(x)=\sqrt{\alpha^{2}+\frac{\alpha \beta L}{D}+\beta^{2}}$

$\alpha=\frac{6000}{\sqrt{2} \times w \times L}$

$\beta=Q D / J$

$D=\frac{1}{2} \sqrt{L^{2}+(w+d)^{2}}$

$J=2 \sqrt{2} \times w \times L\left[\frac{L^{2}}{12}+\frac{(w+d)^{2}}{4}\right]$

$Q=6000 \times\left(14+\frac{L}{2}\right)$

By the HIA, the mini value was found is $f\left(x_{*}\right)=1.72485231$ (error range is less than $3 \%$ ) in average less than 60 iterations.

All above testing results showed that the HIA algorithm could deal with some type difficult Multi-Object optimization problem、some Engineering optimization problem, etc. Results show that the HIA algorithm can improve the quality of the approximate solutions of optimization and increase the speed of solving problem.

There are still more works need to be done. First, More algorithms should be introduced and more comparative studies need be done: non-dominated sorting genetic algorithm (NSGA-II), strength Pareto evolutionary algorithm (SPEA), etc. Second, special testing problems have respectively distinct local Pareto optimal fronts should be introduced, and, testing problems that have no local Pareto optimal fronts and display an opposite trend should be testing.

\section{CONCLUSION}

For the reason of imitating the spread mechanism of infectious diseases, the HIA algorithm needs the spread transmission way of disease and the incremental iteration movement of the every generation new sources of infection.

At the same time, the concept of effective radius of influence, the infection factors etc. were introduced into the HIA algorithm, and all these concepts indeed increase search speed that found the candidate solution set around the target neighborhood.

The experimental results show that HIA algorithm 
can quickly generate more random candidate source distribution, and can quickly move towards their own local best or worst value.

In the Optimization process, for prevent run into partial optimization, the HIA keep on generating new sources of infection of new generation by iteration method, so that HIA algorithm has ability to prevent fall into local optimum.

At the same time, most of the search operations were still near the currently global optimum; The HIA algorithm has good convergence and can quick search to the global optimal solution with fewer times of iterations.

For some above optimization instances, the HIA algorithm has faster speed for search better approximate solution or search the best solution more precisely.

\section{REFERENCES}

[1] Papadimitriou C H, Yannakakis M. Optimization, approximation, and complexity classes $[\mathrm{J}]$. Journal of computer and system sciences, 1991, 43(3): 425-440.

[2] Polëiìak B T. Introduction to optimization[M]. Optimization Software, Publications Division (New York), 1987.

[3] Lourenço H R, Martin O C, Stützle T. Iterated local search: Framework and applications[M] // Handbook of Metaheuristics. Springer US, 2010: 363-397.

[4] Laumanns M, Rudolph G, Schwefel H P. Mutation Control and Convergence in Evolutionary Multi-Object Optimization[J]. HT014601767, 2001.

[5] Socha K, Dorigo M. Ant colony optimization for continuous domains[J]. European Journal of Operational Research, 2008, 185(3): 1155-1173.

[6] Yang X S. Firefly algorithm, stochastic test functions and design optimisation[J]. International Journal of Bio-Inspired Computation, 2010, 2(2): 78-84.

[7] Yang X S. Bat algorithm for multi-objective optimisation[J]. International Journal of Bio-Inspired Computation, 2011, 3(5): 267-274.

[8] Yang X S, Gandomi A H. Bat algorithm: a novel approach for global engineering optimization[J]. Engineering Computations, 2012, 29(5): 464-483.
[9] Yang X S, Deb S. Engineering optimisation by cuckoo search[J]. International Journal of Mathematical Modelling and Numerical Optimisation, 2010, 1(4): 330-343.

[10] Yang X S. Review of meta-heuristics and generalised evolutionary walk algorithm $[\mathrm{J}]$. International Journal of Bio-Inspired Computation, 2011, 3(2): 77-84.

[11] Yang X S. Review of meta-heuristics and generalised evolutionary walk algorithm $[\mathrm{J}]$. International Journal of Bio-Inspired Computation, 2011, 3(2): 77-84.

[12] Yang X S. Metaheuristic algorithms for inverse problems[J]. International Journal of Innovative Computing and Applications, 2013, 5(2): 76-84.

[13] Kirkpatrick S, Jr. D G, Vecchi M P. Optimization by simmulated annealing[J]. science, 1983, 220(4598): 671-680.

[14] Kennedy J, Eberhart R C. A discrete binary version of the particle swarm algorithm[C]//Systems, Man, and Cybernetics, 1997. Computational Cybernetics and Simulation., 1997 IEEE International Conference on. IEEE, 1997, 5: 4104-4108.

[15] Socha K, Dorigo M. Ant colony optimization for continuous domains[J]. European Journal of Operational Research, 2008, 185(3): 1155-1173

[16] Mahdavi M, Fesanghary M, Damangir E. An improved harmony search algorithm for solving optimization problems[J]. Applied mathematics and computation, 2007, 188(2): 1567-1579.

[17] Yang X S, Deb S. Cuckoo search via Lévy flights[C]//Nature \& Biologically Inspired Computing, 2009. NaBIC 2009. World Congress on. IEEE, 2009: 210-214.

[18] Yang X S. Review of meta-heuristics and generalised evolutionary walk algorithm[J]. International Journal of Bio-Inspired Computation, 2011, 3(2): 77-84.

[19] Murray J D. Mathematical biology[M]. springer, 2002.

[20] Štěpán J, Hlubinka D. Kermack-McKendrick epidemic model revisited[J]. Kybernetika, 2007, 43(4): 395-414.

[21] Dagon D, Zou C C, Lee W. Modeling Botnet Propagation Using Time Zones[C]//NDSS. 2006, 6: 2-13.

[22] Boccara N, Cheong K. Automata network SIR models for the spread of infectious diseases in populations of moving individuals $[\mathrm{J}]$. Journal of Physics A: Mathematical and General, 1992, 25(9): 2447.

[23] Brauer F. Some simple epidemic models[J]. Mathematical Biosciences and Engineering, 2006, 3(1): 1.

[24] Cagnina L C, Esquivel S C, Coello C A C. Solving Engineering Optimization Problems with the Simple Constrained Particle Swarm Optimizer[J]. Informatica (Slovenia), 2008, 32(3): 319-326.

[25] Deb K. Optimal design of a welded beam via genetic algorithms[J]. AIAA journal, 1991, 29(11): 2013-2015. 